\title{
106. Hydrogenation of Ethylene with Nickel as a Catalyst at Low Temperatures.
}

\author{
By Osamu Toyama. \\ Chemical Institute, Kyoto Imperial University. \\ (Comm. by Y. OSAKA, M.I.A., Oct. 12, 1935.)
}

Under Prof. S. Horiba's continued guidance the kinetics of the heterogeneous catalysis has been studied in the Laboratory of Physical Chemistry $^{1)}$ for the purpose of the elucidation of its mechanism. The researches have hitherto been made chiefly on reactions having one reactant because the processes involved in the catalysis were generally quite complicated. The author studied the hydrogenation of ethylene from the following two reasons : first, it belongs to one of the simplest of reactions with two reactants, and secondly, it represents one of the fundamentals of the hydrogenation of the unsaturated organic compounds.

Although the catalytic hydrogenation of ethylene has already been variously studied, yet its kineties has not been fully investigated. The present investigation was carried out in the presence of nickel, the most common catalyst to the hydrogenation, at rather low temperatures $0^{\circ}$ to $-78^{\circ} \mathrm{C}$., for studies hitherto made have shown that the reaction at high temperatures above $100^{\circ} \mathrm{C}$. more or less resembled that in a gaseous system, and that consequently it would not reveal clearly any characteristics of the catalysis. Nickel with stable activity was prepared by the reduction at high temperatures $450^{\circ}-500^{\circ} \mathrm{C}$. of the oxide, which was obtained by the calcination of the nitrate at about $400^{\circ} \mathrm{C}$. The rate of reaction was measured statically in two ways: first at constant volume as usual, and then at constant partial pressure of hydrogen or ethylene. The results may be summarised as follows:

(1) The effects of the pretreatment of the catalyst with hydrogen, ethylene, and ethane in the presence of a large quantity of nickel were thus: the treatment with hydrogen favours the initial rate of reaction, while that with ethylene retards it, and that with ethane exerts scarcely any influence.

(2) The relation between the concentrations of the reactants and the reaction velocity was thus: the reaction velocity increases with the increase of hydrogen pressure, but decreases gradually with the increase of ethylene pressure. Accordingly, when hydrogen is in excess, the observed pressure-time curve becomes almost linear, and when ethylene is in excess, the curve gradually approaches to that of a first order reaction with respect to hydgrogen, although the first order is not exactly attained. This relation reveals itself much clearer when the partial pressure of one reactant is kept constant in the course of the reaction; at constant hydrogen pressure, the rate of reaction increases

1) S. Horiba and T. Ri: Rev. Phys. Chem. Japan, 4, 73 (1930); T. Ri : ibid., 5, 41 (1931); M. Kubokawa: ibid., 6, 81 (1932); 8, 18, 33 (1933). 
as the reaction proceeds and reaches a maximum near the end of the reaction; at constant ethylene pressure the rate of reaction decreases quite rapidly and, when the constant pressure of ethylene is high, almost in proportion to hydrogen pressure.

(3) The rate of reaction is almost indifferent to the concentration of ethane in the gas phase, for the addition of ethane to the mixture of the reactants before the reaction brings scarcely any change in the velocity.

(4) The similarity of the curves at $-78^{\circ} \mathrm{C}$ to those at $0^{\circ} \mathrm{C}$ indicates that the reaction takes place in this temperature range according to the same mechanism. The apparent heat of activation is about $6 \mathrm{Kcal}$.

All these experimental results can be best interpreted by the following reaction mechanism : the combination of ethylene and hydrogen in the presence of nickel is a bimolecular reaction on the surface of the catalyst; the surface reaction represents the slowest process of all involved in the mechanism and determines the reaction velocity of the system; as both reactants are adsorbed on the same active surface of the catalyst, there is a competition for the active surface between them with the result that the concentration of hydrogen on the active surface is markedly decreased by ethylene which is adsorbed on the active surface more strongly than hydrogen; the rate of reaction, therefore, depends chiefly upon the surface concentration of hydrogen, which is increased by the pressure increase of hydrogen, but decreased by that of ethylene; ethane, the product, has little effect on the rate of reaction, for it is only slightly adsorbed on the active surface.

Hence, the rate of reaction is represented by the following equation:

$$
\frac{d x}{d t}=\text { const. } \sigma_{H} \sigma_{E},
$$

where $\sigma_{H}$ and $\sigma_{E}$ are the surface concentrations of hydrogen and ethylene respectively. It is assumed that the concentrations of both reactants on the active surface are approximately equal to those in an adsorption equilibrium, whence $\sigma$ 's are given by Langmuir's isotherm as follows :

$$
\begin{gathered}
\sigma_{H}=\text { const. } \frac{p_{H}}{1+\alpha p_{H}+\beta p_{E}}, \\
\sigma_{E}=\text { const. } \frac{p_{H}}{1+\alpha p_{H}+\beta p_{E}},
\end{gathered}
$$

where $p_{H}$ and $p_{E}$ are the pressures of hydrogen and ethylene in the gas phase $; \alpha$ and $\beta$ the adsorption coefficients of hydrogen and ethylene respectively ; $\beta$ must be greater than $\alpha$ since ethylene is adsorbed on the active surface more strongly than hydrogen. The rate of reaction is now expressed as follows:

$$
\frac{d x}{d t}=k \frac{p_{H} p_{E}}{\left(1+\alpha p_{H}+\beta p_{E}\right)^{2}}
$$

It has been ascertained that the velocity equation thus obtained explains the experimental results quite well, proving that the above-mentioned 
mechanism is adequate, and the possiblities of such a mechanism as a chain reaction in the gas phase or a collision between one adsorbed reactant and the other in the gas phase, must be denied from various considerations.

The results obtained by Pease ${ }^{1)}$ on the same reaction with copper as catalyst may be explained by the same mechanism as above adopted. But zur Strassen's recent work ${ }^{2}$ with a nickel ribbon as catalyst had a different feature and his results are to be interpreted by the assumption that both reactants have different adsorption surfaces.

1) R. N. Pease: J. Am. Chem. Soc., 45, 1196, 2235 (1923) ; 49, 2503 (1927).

2) H. zur Strassen: Z. physik. Chem. (A), 169, 81 (1934). 